\title{
Increase in plasma adrenomedullin in patients with heart failure characterised by diastolic dysfunction
}

\author{
C-M Yu, B M Y Cheung, R Leung, Q Wang, W-H Lai, C-P Lau
}

\begin{abstract}
Objective-To investigate the relation between plasma adrenomedullin and the severity of diastolic dysfunction in patients with heart failure.

Design-Prospective study.

Setting-University teaching hospital.

Patients-77 patients (mean (SEM) age 66.3 (1.2) years; $75 \%$ male) who were being followed in the outpatient clinic after admission to hospital for acute heart failure.

Interventions-Same day echocardiography with Doppler studies; determination of venous adrenomedullin concentration by radioimmunoassay.

Main outcome measures-Plasma adrenomedullin concentration and its correlation with systolic and diastolic function.

Results-31 patients (40\%) had isolated diastolic dysfunction (ejection fraction $>50 \%$ ), and the remaining 46 had a depressed ejection fraction $(<50 \%)$. Of the patients with diastolic dysfunction, 17 had a restrictive filling pattern. In all but one of these there was coexisting systolic failure $\left(\chi^{2}=10.7, p=0.001\right)$. Patients with systolic heart failure and a restrictive filling pattern (group $1, \mathrm{n}=16$ ) had a higher plasma adrenomedullin than those with systolic failure and a non-restrictive filling pattern (group $2, \mathrm{n}=30$ ) or with isolated diastolic heart failure and a nonrestrictive filling pattern (group 3, $\mathrm{n}=30$ ) (mean (SEM): 91.7 (21.1) v 38.4 (8.8) v 34.0 (6.5) $\mathrm{pmol} / \mathrm{l}$, both $\mathrm{p}<0.05)$. All heart failure values were higher $(\mathrm{p}<0.01)$ than the control value (6.9 (1.2) pmol/1). Ejection fraction and left ventricular dimensions were similar in groups 1 and 2. Plasma adrenomedullin did not correlate with ejection fraction or New York Heart Association functional class. Stepwise multiple regression analysis showed that the presence of a restrictive filling pattern was the only independent variable associated with a high plasma adrenomedullin. Conclusions-Plasma adrenomedullin concentrations in patients with heart failure are determined by the presence of diastolic dysfunction, and are especially raised in the presence of a restrictive filling pattern. There appears to be no correlation with systolic dysfunction.
\end{abstract}

(Heart 2001;86:155-160)

Keywords: adrenomedullin; heart failure; diastolic dysfunction; Doppler echocardiography

Human adrenomedullin is a 52 amino acid peptide which shows partial sequence homology with the potent vasodilator calcitonin related gene peptide. ${ }^{1}$ It was first isolated from phaeochromocytoma and the adrenal medulla, ${ }^{1}$ but it is also found in the heart, lung, and kidney. ${ }^{2}$ It is present in the plasma, suggesting that it may be a circulating hormone. $^{3}$ Although its physiological role is unclear, animal studies have shown that it lowers systemic vascular resistance and that it is natriuretic and diuretic. ${ }^{4}$

Plasma adrenomedullin is increased in several diseases. ${ }^{5}$ Concentrations are raised in congestive heart failure and there is a weak inverse correlation with left ventricular ejection fraction. ${ }^{6-8}$ However, in patients with heart failure there is often coexisting left ventricular diastolic dysfunction, and this can be assessed readily and non-invasively by Doppler echocardiography. ${ }^{9}$ Other vasodilator neuropeptides that are increased in heart failure-such as atrial and brain natriuretic peptides - show a greater increase in patients with restrictive diastolic filling, which is the most severe form of diastolic dysfunction. ${ }^{10} \mathrm{An}$ increase in these vasodilator peptides was thought to compensate for the fluid overload and ventricular chamber distension that are characteristic of heart failure. However, no attempt has been made to determine whether elevation of adrenomedullin in heart failure is related to diastolic dysfunction. Our objective in the present study was therefore to investigate the relation between plasma adrenomedullin concentrations and cardiac function in patients with heart failure, and in particular whether raised adrenomedullin is related to systolic or diastolic dysfunction.

\section{Methods}

PATIENTS

We studied 77 patients (mean (SEM) age, 66.3 (1.2) years; $75 \%$ male) with clinical heart failure and in New York Heart Association (NYHA) class II-IV. They had symptoms of fatigue and exertional dyspnoea, orthopnoea, or paroxysmal nocturnal dyspnoea, with or without oedema, and there were clinical signs of fluid overload (crepitations over the chest and elevation of the jugular venous pressure, with or without pitting ankle oedema). The patients were categorised as having systolic heart failure (left ventricular ejection fraction 
$\leqslant 50 \% ; n=46)$ or isolated diastolic heart failure with preserved systolic function (left ventricular ejection fraction $>50 \% ; n=31$ ). The causes of the heart failure were coronary heart disease $(86 \%)$, idiopathic dilated cardiomyopathy (9\%), hypertensive heart disease $(3 \%)$, alcoholic cardiomyopathy $(1 \%)$, and valvar heart disease $(1 \%)$. Among the patients with coronary heart disease, 32 had evidence of myocardial infarction and 32 also had coexisting hypertension.

Coronary angiography was performed in 36 patients with coronary heart disease and in all the patients with dilated cardiomyopathy.

Patients with significant major organ dysfunction (for example, renal, hepatic, and respiratory disorders) which might affect the adrenomedullin assay ${ }^{5}$ were excluded. Seventeen normal healthy subjects of similar age (61.4 (1.8) years) and sex (70\% male) were included as controls.

The study protocol was approved by the faculty ethics committee, and informed consent was obtained from all subjects.

\section{ECHOCARDIOGRAPHY}

Echocardiography was performed on the same day that blood was taken for plasma adrenomedullin. Standard cross sectional echocardiography with $M$ mode assessment of systolic function was done following the guidelines of the American Society of Echocardiography. ${ }^{11}$ Pulsed Doppler assessment of diastolic function was performed by interrogation of the flow velocity profiles at the mitral annulus, as previously described, ${ }^{12}{ }^{13}$ and it was confirmed by assessment of the pulmonary venous inflow profile if necessary. ${ }^{14}$ The diastolic variables measured included peak early diastolic filling velocity ( $E$ velocity), the deceleration time of the $\mathrm{E}$ wave, peak atrial filling velocity (A velocity), the ratio of early to atrial peak filling velocities (E:A ratio), and the isovolumic relaxation time. At least three consecutive beats were measured and the average values taken.

Patients were classified as having left ventricular diastolic dysfunction according to the following criteria:

- a restrictive filling pattern was defined as an $\mathrm{E}: \mathrm{A}$ ratio of $\geqslant 2$ or $\mathrm{E}: \mathrm{A}=1$ to 2 and deceleration time of < $140 \mathrm{~ms}$;

- an abnormal relaxation pattern was defined as an $\mathrm{E}: \mathrm{A}$ ratio of $<1$ or $\mathrm{E}: \mathrm{A}$ ratio $=1$ to 2 and deceleration time of $>240 \mathrm{~ms}$;

- a pseudonormal pattern was defined as a normal transmitral pattern but an abnormal pulmonary venous flow profile (reversed systolic to diastolic forward flow ratio $)^{12-14}$;

- where there was abnormal relaxation together with a pseudonormal pattern, this was collectively called a non-restrictive filling pattern.

MEASUREMENT OF PLASMA ADRENOMEDULLIN Assay of plasma adrenomedullin has been described previously. ${ }^{5}{ }^{15}$ Venous blood $(6 \mathrm{ml})$ was chilled in EDTA tubes on ice. Samples were promptly centrifuged and the plasma stored at $-40^{\circ} \mathrm{C}$ until the time of assay. The plasma immunoreactivity of adrenomedullin was measured using a commercially available radioimmunoassay kit (Peninsula Laboratories, Belmont, California, USA). Briefly, $3 \mathrm{ml}$ plasma samples were deproteinated with $0.75 \mathrm{ml}$ of $2 \mathrm{M}$ hydrochloric acid. After centrifugation for 10 minutes at $2000 \mathrm{~g}$, the supernatants were loaded onto Sep-Pak C18 cartridges (Waters Associates, Milford, Massachusetts, USA), which had been activated with $100 \%$ methanol and double distilled deionised water. The cartridges were then washed twice with $5 \mathrm{ml} 0.1 \%$ trifluoroacetic acid and eluted with $60 \%$ acetonitrile in $0.1 \%$ trifluoroacetic acid. The eluates were freeze dried overnight and resuspended in $250 \mu \mathrm{l}$ radioimmunoassay buffer. A $100 \mu \mathrm{l}$ aliquot of standard adrenomedullin or assay sample was incubated overnight at $4^{\circ} \mathrm{C}$ with $100 \mu \mathrm{l}$ of rabbit antiadrenomedullin antiserum. Then $100 \mu \mathrm{l}$ of ${ }^{125} \mathrm{I}-$ adrenomedullin was added to each tube and incubated for a further 24 hours. Antibody bound adrenomedullin was precipitated using a goat antirabbit antiserum and measured in a gamma counter.

The detection limit (2 SD from zero) was $1 \mathrm{pg} /$ tube or $0.14 \mathrm{pM}$ adrenomedullin in plasma. All samples, including those from controls, were above the limit of detection. The intra-assay and interassay coefficients of variation were $7 \%$ and $12 \%$, respectively. Recovery of adrenomedullin after extraction was determined by adding labelled adrenomedullin to plasma before extraction and was found to be $72 \%$. All values are quoted without correction for extraction efficiency.

\section{STATISTICS}

Data were analysed using a statistical software program (SPSS for Windows, version 7.5; SPSS Inc, Chicago, Illinois, USA). Mean plasma adrenomedullin concentrations between different groups were compared using the unpaired $t$ test. Stepwise multiple regression analysis was performed with plasma adrenomedullin concentration as the dependent variable and indices of systolic and diastolic function as the independent (predictor) variables, with age, sex, and heart rate as covariates. Data are expressed as mean (SEM). A two tailed $p$ value $<0.05$ was considered significant.

\section{Results}

The mean (SEM) left ventricular ejection fraction in these 77 patients was 51.0 (1.7)\%. Demographic and clinical variables in the patients with systolic and isolated diastolic heart failure are given in table 1. NYHA functional class distribution was class II in $77 \%$, class III in $21 \%$, and class IV in $2 \%$. There was no significant correlation between plasma adrenomedullin concentration and the severity of heart failure symptoms (NYHA class II: plasma adrenomedullin $45.6 \quad$ (8.6) pmol/1; class III/IV: plasma adrenomedullin 59.9 (13.6) pmol/1) (NS). 
Table 1 Clinical features in patients with systolic and isolated diastolic heart failure

\begin{tabular}{|c|c|c|}
\hline & $\begin{array}{l}\text { Systolic } \\
\text { heart failure } \\
(n=46)\end{array}$ & $\begin{array}{l}\text { Isolated } \\
\text { diastolic heart } \\
\text { failure }(n=31)\end{array}$ \\
\hline Age (years) & $65.7(1.8)$ & $66.5(1.5)$ \\
\hline $\operatorname{Sex}(M: F)(\%)$ & $72: 28$ & $81: 19$ \\
\hline \multicolumn{3}{|l|}{ NYHA class (\%) } \\
\hline II & 77.1 & 83.3 \\
\hline III & 20.0 & 16.7 \\
\hline IV & 2.9 & - \\
\hline \multicolumn{3}{|l|}{ Aetiology of heart failure } \\
\hline Ischaemic heart disease & 84.1 & 87.9 \\
\hline Dilated cardiomyopathy & 9.1 & 9.1 \\
\hline Hypertension & 4.5 & - \\
\hline Valvar heart disease & - & 3.0 \\
\hline Alcoholic cardiomyopathy & 2.3 & - \\
\hline \multicolumn{3}{|l|}{ Drugs } \\
\hline Diuretics & 71.4 & 60.6 \\
\hline ACE inhibitors & 76.2 & 81.8 \\
\hline$\beta$ Blockers & 21.1 & 38.9 \\
\hline Nitrates & 69.4 & 71.4 \\
\hline Calcium channel blockers & 14.3 & 23.1 \\
\hline Lipid lowering agents & 47.6 & 66.7 \\
\hline Aspirin & 68.2 & 78.8 \\
\hline Digoxin & 14.3 & 3.1 \\
\hline LV diameter, diastole $(\mathrm{cm})$ & $6.1(0.2)$ & $5.1(0.1)^{\star}$ \\
\hline LV diameter, systole $(\mathrm{cm})$ & $5.0(0.2)$ & $3.6(0.1)^{\star}$ \\
\hline Fractional shortening (\%) & $16.5(0.6)$ & $29.8(1.1)^{\star}$ \\
\hline $\operatorname{LVEF}(\%)$ & $40.3(1.3)$ & $65.5(1.6)^{\star}$ \\
\hline \multicolumn{3}{|l|}{ Diastolic function (\%) } \\
\hline RFP & 36.4 & $3.0 \dagger$ \\
\hline Non-RFP & 63.6 & 97.0 \\
\hline Plasma adrenomedullin (pmol/1) & $57.0(9.9)$ & $33.4(6.1)$ \\
\hline
\end{tabular}

Values are mean (SEM) or \%.

${ }^{\star} \mathrm{p}<0.001 v$ patients with systolic and isolated diastolic heart failure.

$t \chi^{2}=10.7, \mathrm{p}=0.001 v$ patients with systolic and isolated diastolic heart failure.

ACE, angiotensin converting enzyme; LV, left ventricular; LVEF, left ventricular ejection fraction; NYHA, New York Heart Association; RFP, restrictive filling pattern.

PLASMA ADRENOMEDULLIN AND DIASTOLIC

FUNCTION

Seventeen patients had diastolic function with a restrictive filling pattern, while the other 60 patients had a non-restrictive filling pattern. In the non-restrictive group, 46 had an abnormal relaxation pattern, 11 had a pseudonormal pattern, and three had a normal pattern. When the prevalence of left ventricular diastolic dysfunction was compared among patients with systolic and isolated diastolic heart failure, it was found that all but one of the patients with a restrictive filling pattern had concomitant systolic heart failure (restrictive filling pattern in systolic $v$ diastolic heart failure, $36 \% v 3 \%$; $\chi^{2}=10.7, \mathrm{p}=0.001$ ).

The mean plasma adrenomedullin concentration in the heart failure patients was 47.5 (6.5) pmol/1, significantly higher than the mean value in the normal controls $(6.9$ (1.2) pmol/1) ( $\mathrm{p}<0.01$; fig 1). Among the heart failure patients, 16 had systolic heart failure and a restrictive filling pattern (group 1), 30 had systolic heart failure and a non-restrictive filling pattern (group 2), and 30 had isolated diastolic heart failure and a non-restrictive filling pattern (group 3). It was found that patients in group 1 had a higher plasma adrenomedullin concentration than those in either group 2 or group $3(91.7(21.1) v 38.4$ (8.8) v 34.0 (6.5) pmol/1; both $\mathrm{p}<0.05)$, though the values did not differ between groups 2 and 3. However, the plasma adrenomedullin concentrations were higher than the control value $(p<0.01)$ in all three heart failure groups.

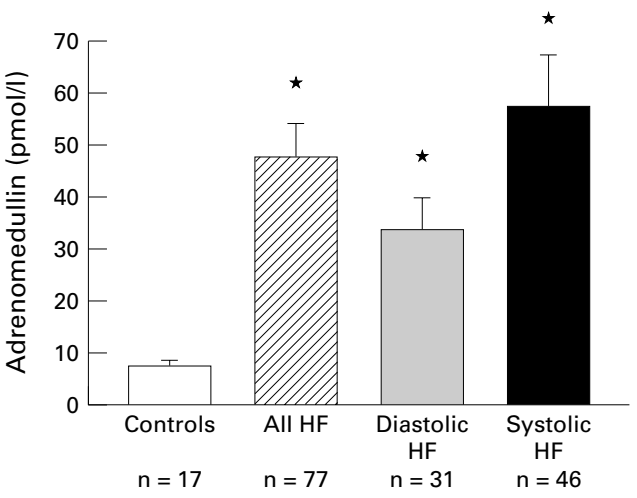

Figure 1 Mean plasma adrenomedullin concentrations in controls (white bar), all heart failure patients (HF) (hatched bar), patients with isolated diastolic heart failure (grey bar), and patients with systolic heart failure (black bar). Error bars $=S E M .{ }^{\star} p<0.01$ v controls.
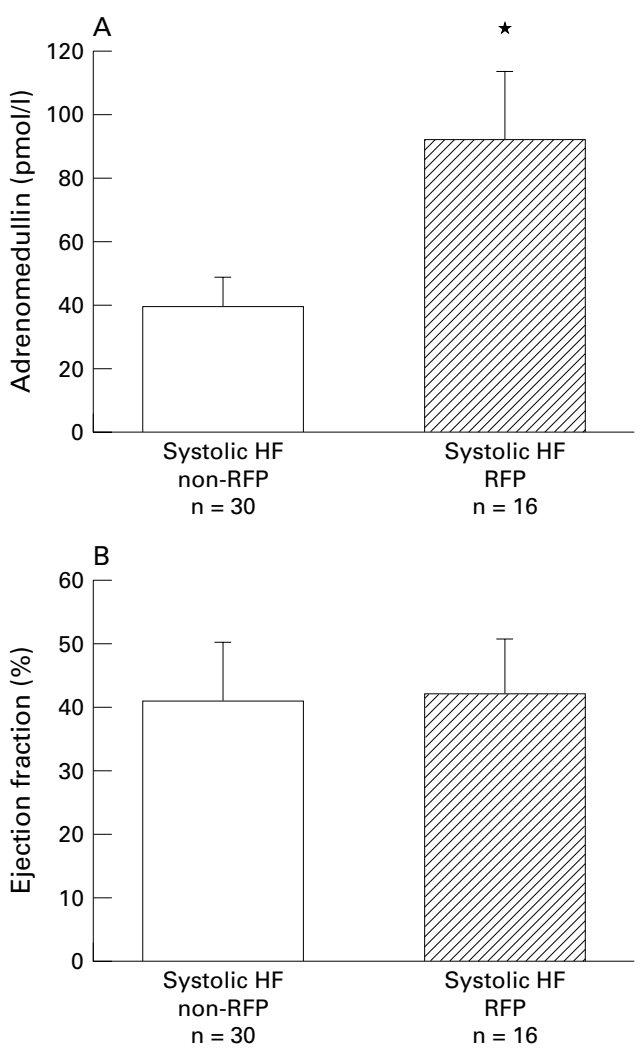

Figure 2 Effect of diastolic dysfunction with restrictive filling pattern (RFP) on plasma adrenomedullin concentrations in patients with systolic heart failure $(H F)$. (A) Patients with RFP (hatched bar) had significantly higher plasma adrenomedullin that those with a non-restrictive pattern (non-RFP) (white bar). ${ }^{\star} p<0.05$, $R F P$ v non-RFP groups. (B) There was no difference in the left ventricular ejection fraction between RFP and non-RFP groups.

There was no difference in the ejection fraction between groups 1 and $2(40.9$ (9.2) $v 42.0$ (8.6)\%; NS) (fig 2). Similarly, patients with systolic heart failure and a concomitant abnormal diastolic relaxation pattern also had a lower plasma adrenomedullin concentration than the patients in group 1 (36.3 (10.4) v 91.7 (21.1) $\mathrm{pmol} / \mathrm{l} ; \mathrm{p}<0.05)$ but no difference in the ejection fraction. The left ventricular end diastolic dimension $(6.2(0.2) v 6.0(0.2) \mathrm{cm})$ and end systolic dimension (5.1 (0.2) $v 5.0$ $(0.2) \mathrm{cm}$ ) did not differ between groups 1 and 
Table 2 Correlation between individual systolic and diastolic variables, clinical severity of heart symptoms, and plasma adrenomedullin concentrations

\begin{tabular}{|c|c|c|c|c|c|c|c|c|}
\hline \multirow[b]{2}{*}{ Variable } & \multicolumn{2}{|c|}{ All patients } & \multicolumn{2}{|c|}{$\begin{array}{l}\text { Systolic heart failure } \\
\text { with RFP }\end{array}$} & \multicolumn{2}{|c|}{$\begin{array}{l}\text { Systolic heart failure } \\
\text { with non-RFP }\end{array}$} & \multicolumn{2}{|c|}{ Diastolic heart failure } \\
\hline & $r$ & $p$ Value & $r$ & $p$ Value & $r$ & $p$ Value & $r$ & $p$ Value \\
\hline E velocity & 0.25 & $<0.05$ & 0.52 & $<0.005$ & 0.04 & NS & 0.003 & NS \\
\hline A velocity & -0.13 & NS & -0.02 & NS & 0.03 & NS & 0.32 & NS \\
\hline E:A ratio & 0.33 & 0.005 & 0.35 & $<0.05$ & 0.07 & NS & -0.22 & NS \\
\hline Deceleration time of $\mathrm{E}$ & -0.13 & NS & 0.19 & NS & -0.02 & NS & 0.43 & $<0.05$ \\
\hline Isovolumic relaxation time & 0.01 & NS & -0.02 & NS & 0.27 & NS & -0.10 & NS \\
\hline End diastolic dimension & 0.25 & $<0.05$ & 0.10 & NS & 0.18 & NS & 0.21 & NS \\
\hline End systolic dimension & 0.21 & NS & 0.09 & NS & 0.20 & NS & 0.18 & NS \\
\hline Fractional shortening & -0.17 & NS & -0.02 & NS & -0.21 & NS & 0.04 & NS \\
\hline LVEF by $\mathrm{M}$ mode & -0.20 & NS & -0.11 & NS & -0.23 & NS & 0.04 & NS \\
\hline LVEF by Simpson's method & -0.15 & NS & -0.06 & NS & -0.10 & NS & -0.19 & NS \\
\hline Left atrial size & 0.18 & NS & 0.26 & NS & 0.03 & NS & 0.02 & NS \\
\hline PASP & 0.03 & NS & -0.22 & NS & -0.05 & NS & 0.13 & NS \\
\hline NHYA class & 0.14 & NS & 0.21 & NS & 0.29 & NS & 0.27 & NS \\
\hline Diuretics used & 0.12 & NS & 0.30 & NS & -0.16 & NS & -0.28 & NS \\
\hline
\end{tabular}

A velocity, transmitral peak atrial filling velocity; E velocity, transmitral peak early filling velocity; E:A ratio, ratio of transmitral early to atrial peak filling velocity; LVEF, left ventricular ejection fraction; NYHA, New York Heart Association; PASP, pulmonary arterial systolic pressure; RFP, restrictive filling pattern of diastolic dysfunction.

2 , but both values were larger $(p=0.001)$ than in group 3.

The correlations between plasma adrenomedullin and the individual diastolic variables in all three groups are shown in table 2. We found that the transmitral E:A ratio, the $\mathrm{E}$ velocity, and the left ventricular end diastolic dimension were positively correlated with plasma adrenomedullin. Among the patients in group 1, transmitral E:A ratio and E velocity were positively correlated with plasma adrenomedullin, while in group 3 the deceleration time was the best predictor of plasma adrenomedullin.

To investigate the relation between the severity of diastolic dysfunction, NYHA class, and plasma adrenomedullin concentration in patients with systolic heart failure, these variables were compared in between groups 1 and 2. It was found that group 1 contained more patients in NYHA class III or IV than in class II $\left(46.7 \% v 14.3 \% ; \chi^{2}=4.6 ; \mathrm{p}<0.05\right)$. However, a higher NYHA class itself did not predict a higher plasma adrenomedullin concentration (class I/II, 55.0 (14.0) pmol/1 $v$ class

Table 3 Stepwise multiple regression analysis comparing the correlation between individual variables and plasma adrenomedullin concentration

\begin{tabular}{lll}
\hline Variable & $\begin{array}{l}\text { Regression } \\
\text { coefficient }\end{array}$ & p Value \\
\hline RFP $v$ non-RFP & 51.4 & 0.02 \\
E velocity & -0.15 & NS \\
A velocity & 0.82 & NS \\
E:A ratio & 46.9 & NS \\
Deceleration time of E & 0.11 & NS \\
Isovolumic relaxation time & 0.67 & NS \\
End diastolic dimension & 12.3 & NS \\
End systolic dimension & -1.6 & NS \\
Fractional shortening & 1.13 & NS \\
LVEF by M mode & -0.21 & NS \\
LVEF by Simpson's method & -0.81 & NS \\
Age & 0.41 & NS \\
Sex & 19.9 & NS \\
Heart rate & 0.55 & NS \\
NYHA class & 3.41 & NS \\
Daily diuretic consumption & & NS
\end{tabular}

${ }^{\star}$ Categorical variables.

A velocity, transmitral peak atrial filling velocity; E velocity, transmitral peak early filling velocity; E:A ratio, ratio of transmitral early to atrial peak filling velocity; LVEF, left ventricular ejection fraction; NYHA, New York Heart Association; PASP, pulmonary arterial systolic pressure; RFP, restrictive filling pattern of diastolic dysfunction.
III/IV, 61.3 (22.9) pmol/1 (NS)). Therefore the elevation of plasma adrenomedullin in patients with a restrictive filling pattern cannot be explained by the higher NYHA class.

PLASMA ADRENOMEDULLIN AND SYSTOLIC FUNCTION

There was no difference in plasma adrenomedullin between patients with systolic heart failure and patients with diastolic heart failure (57.0 (9.9) v 33.4 (6.1) pmol/1, NS). Furthermore, there was no correlation between plasma adrenomedullin and left ventricular ejection fraction, measured by either M mode or Simpson's method. Similarly, plasma adrenomedullin did not correlate with fractional shortening (table 2).

Stepwise multiple regression analysis was used to characterise the relation between plasma adrenomedullin and systolic function, diastolic function, and demographic variables (table 3). The only variable identified as being independently associated with plasma adrenomedullin was the presence of diastolic dysfunction characterised by a restrictive filling pattern (table 3).

\section{Discussion}

In this study we found that plasma adrenomedullin was raised in both systolic and isolated diastolic heart failure. Its concentration was determined by the severity of diastolic dysfunction, but not by the degree of systolic failure. In patients with systolic heart failure, a substantial proportion had a restrictive filling pattern, which is associated with higher plasma adrenomedullin concentrations than found in patients with a non-restrictive filling pattern. However, the patients with a restrictive filling pattern had comparable ejection fraction and left ventricular dimensions to those with a non-restrictive filling pattern. The presence of a restrictive filling pattern implies the most severe form of diastolic dysfunction. ${ }^{16}$ However, patients with isolated diastolic heart failure rarely have a restrictive filling pattern, and their plasma adrenomedullin concentrations were similar to those in patients with systolic heart failure and a non-restrictive 
filling pattern, despite a much higher ejection fraction (comparing groups 2 and 3). The importance of diastolic dysfunction as a major determinant of plasma adrenomedullin concentrations was further confirmed by multiple regression analysis. These findings contrast with previous reports which showed a weakly negative correlation between plasma adrenomedullin concentrations and the ejection fraction. ${ }^{6-8}$ However, previous studies have not explored the major confounding effect of diastolic dysfunction. In fact, restrictive diastolic dysfunction is particularly common in patients with left ventricular systolic failure. ${ }^{16}$ As there was no correlation between the ejection fraction and adrenomedullin, our results showed that the presence of a restrictive filling pattern was a more important determinant of the elevation of adrenomedullin than systolic dysfunction.

In the presence of diastolic dysfunction, the filling pressure is known to be increased. With a restrictive filling pattern, left ventricular diastolic pressure is higher than with a non-restrictive pattern, and this has been confirmed by invasive studies. ${ }^{17}$ Therefore it is possible that the raised plasma adrenomedullin found in patients with diastolic dysfunction is primarily related to the increased filling pressure of the heart. As the presence of a restrictive filling pattern may be a marker of more severe heart failure and hence of a worse prognosis, ${ }^{13} 18$ one might argue that the relation between diastolic dysfunction and adrenomedullin concentrations is attributable to other clinical features that accompany severe heart failure. However, despite the fact that patients with systolic heart failure and a restrictive filling pattern were more likely to be in NYHA functional class III and IV than those with a non-restrictive filling pattern, neither NYHA class nor the daily consumption of diuretics correlated with plasma adrenomedullin concentrations. It is therefore likely that the increased circulating adrenomedullin is primarily explained by mechanisms related to the development of diastolic dysfunction - such as the raised filling pressure-rather than to the associated clinical sequelae.

The role of adrenomedullin in heart failure is not fully understood. In animal studies, adrenomedullin infusion lowers the systemic vascular resistance and causes natriuresis. ${ }^{4}$ As increasing circulating concentrations of adrenomedullin reflect more severe diastolic dysfunction, it is tempting to speculate that adrenomedullin may also be part of the neurohormone response to fluid overload in congestive heart failure, as are atrial and brain natriuretic peptides. ${ }^{19}$ This is consistent with a previous report that in patients with systolic heart failure the plasma concentrations of natriuretic peptides are higher in those with a restrictive filling pattern than in those with a non-restrictive pattern ${ }^{10}$ or in those with isolated diastolic dysfunction. ${ }^{20}$ Indeed the plasma concentrations of adrenomedullin and natriuretic peptides are intercorrelated. ${ }^{7}$
Although the site and mechanisms of the increased production of adrenomedullin cannot be determined from the present study, an increase in the expression of adrenomedullin in human ventricular myocytes has been demonstrated in heart failure. ${ }^{6}$ As the pathophysiological changes of a restrictive filling pattern are related to the high diastolic filling pressure of the left ventricle as well as to decreases in ventricular compliance, ${ }^{9}$ the increased tension and stretching of the myocytes may stimulate the local production and secretion of adrenomedullin, as well as of the other natriuretic peptides. ${ }^{21}$ Recently, increased ventricular production of adrenomedullin has been demonstrated in animal models of volume and pressure overloaded cardiac hypertrophy-conditions known to induce diastolic dysfunction. ${ }^{22}$ On the other hand, right ventricular function is unlikely to affect adrenomedullin concentrations, as pulmonary arterial systolic pressure did not differ between groups with different patterns of diastolic dysfunction.

Adrenomedullin is secreted in stress, and cytokines such as interleukin-1 and tumour necrosis factor are known to stimulate its production. ${ }^{23}$ Adrenomedullin may be co-secreted with catecholamines from adrenal medullary cells. ${ }^{24}$ As these factors coexist in heart failure, they may affect the measured plasma concentrations of adrenomedullin. In addition, drug treatments used in heart failure, such as loop diuretics and vasodilators, could modify adrenomedullin concentrations in these patients.

\section{CONCLUSIONS}

Plasma adrenomedullin is increased in patients with systolic and isolated diastolic heart failure, and is particularly raised in the presence of a restrictive filling pattern. However, the adrenomedullin concentrations do not correlate with indices of left ventricular systolic function.

CMY, BMYC, and CPL are members of the Institute of Cardiovascular Science and Medicine, University of Hong Kong. BMYC was in receipt of a Committee for Research and Conference Grant from the University of Hong Kong.

1 Kitamura K, Kangawa K, Kawamoto M, et al. Adrenomedullin: a novel hypotensive peptide isolated from human pheochromocytoma. Biochem Biophys Res Commun 1993;192:553-60.

2 Nishikimi T, Kitamura K, Saito Y, et al. Clinical studies on the sites of production and clearance of circulating adrenomedullin in human subjects. Hypertension 1994;24: 600-4.

3 Ichiki Y, Kitamura K, Kangawa K, et al. Distribution and characterization of immunoreactive adrenomedullin in characterization of immunoreactive adrenomedullin

4 Ishiyama Y, Kitamura K, Ichiki Y, et al. Hemodynamic effects of a novel hypotensive peptide, human adrenomedullin, in rats. Eur $\mathcal{F}$ Pharmacol 1993;241:271-3.

5 Cheung B, Leung R. Elevated plasma levels of human adrenomedullin in cardiovascular, respiratory, hepatic and renal disorders. Clin Sci (Colch) 1997;92:59-62.

6 Jougasaki M, Wei CM, McKinley LJ, et al. Elevation of circulating and ventricular adrenomedullin in human congestive heart failure. Circulation 1995;92:286-9.

7 Kato J, Kobayashi K, Etoh T, et al. Plasma adrenomedullin concentration in patients with heart failure. F Clin Endocrinol Metab 1996;81:180-3.

8 Nishikimi T, Saito Y, Kitamura K, et al. Increased plasma levels of adrenomedullin in patients with heart failure. $7 \mathrm{Am}$ Coll Cardiol 1995;26:1424-31.

9 Cohen GI, Pietrolungo JF, Thomas JD, et al. A practical guide to assessment of ventricular diastolic function using Doppler echocardiography. F Am Coll Cardiol 1996;27: 1753-60. 
10 Yu CM, Sanderson JE, Shum IO, et al. Diastolic dysfunction and natriuretic peptides in systolic heart failure. Higher ANP and BNP levels are associated with the restrictive filling pattern. Eur Heart f 1996;17:1694-702.

11 Sahn DJ, DeMaria A, Kisslo J, et al. Recommendations regarding quantitation in M-mode echocardiography: results of a survey of echocardiographic measurements. Circulation 1978;58:1072-83.

$12 \mathrm{Yu}$ CM, Sanderson JE, Chan S, et al. Right ventricular diastolic dysfunction in heart failure. Circulation 1996;93 1509-14.

13 Xie GY, Berk MR, Smith MD, et al. Prognostic value of Doppler transmitral flow patterns in patients with congestive heart failure. $\mathcal{F}$ Am Coll Cardiol 1994;24:132-9.

14 Rossvoll O, Hatle LK. Pulmonary venous flow velocities recorded by transthoracic Doppler ultrasound: relation to left ventricular diastolic pressures. 7 Am Coll Cardiol 1993 21:1687-96.

15 Lewis LK, Smith MW, Yandle TG, et al. Adrenomedullin(152) measured in human plasma by radioimmunoassay: 52) measured in human plasma by radioimmunoassay: plasma concen

16 Pinamonti B, Di Lenarda A, Sinagra G, et al. Restrictive left ventricular filling pattern in dilated cardiomyopathy assessed by Doppler echocardiography: clinical, echocardiographic and hemodynamic correlations and prognostic implications. Heart Muscle Disease Study Group. $\mathcal{F} A m$ Coll Cardiol 1993;22:808-15.

17 Appleton CP, Hatle LK, Popp RL. Relation of transmitra flow velocity patterns to left ventricular diastolic function new insights from a combined hemodynamic and Doppler echocardiographic study. 7 Am Coll Cardiol 1988;12:426-

18 Yu CM, Sanderson JE. Different prognostic significance of right and left ventricular diastolic dysfunction in heart failure. Clin Cardiol 1999;22:504-12.

19 Wei CM, Heublein DM, Perrella MA, et al. Natriuretic peptide system in human heart failure. Circulation 1993;88: 1004-9.

20 Lang CC, Prasad N, McAlpine HM, et al. Increased plasma levels of brain natriuretic peptide in patients with isolated diastolic dysfunction. Am Heart f 1994;127:1635-6.

21 Yasue $\mathrm{H}$, Yoshimura $M$, Sumida $\mathrm{H}$, et al. Localization and mechanism of secretion of B-type natriuretic peptide in comparison with those of A-type natriuretic peptide in normal subjects and patients with heart failure. Circulation 1994;90:195-203.

22 Yoshihara F, Nishikimi T, Horio $\mathrm{T}$, et al. Ventricular adrenomedullin concentration is a sensitive biochemical marker for volume and pressure overload in rats. $A m \mathcal{F}$ Physiol Heart Circ Physiol 2000;278:H633-42.

23 Sugo S, Minamino N, Shoji H, et al. Interleukin-1, tumor necrosis factor and lipopolysaccharide additively stimulate production of adrenomedullin in vascular smooth muscle production of adrenomedullin in vascular smooth m
cells. Biochem Biophys Res Commun 1995;207:25-32.

24 Katoh F, Niina H, Kitamura K, et al. $\mathrm{Ca}(2+)$-dependent cosecretion of adrenomedullin and catecholamines mediated by nicotinic receptors in bovine cultured adrenal medullary cells. FEBS Lett 1994;348:61-4.

\section{IMAGES IN CARDIOLOGY}

\section{Pericardial constriction secondary to calcified intrapericardial haematoma}

In 1993, a 56 year old man was involved in a road traffic accident where his car was struck violently by another vehicle. Despite wearing a seatbelt he developed severe chest pain. At his local casualty department a chest radiograph confirmed a fractured sternum but was otherwise normal. An ECG showed $\mathrm{T}$ wave inversion in leads V1 to V6. Creatinine kinase concentrations were raised. A diagnosis of acute cardiac contusion was made and treated conservatively.

Some years later he developed signs of pericardial constriction. Contrast enhanced computed tomography (right) revealed a right pleural effusion in addition to a $6 \mathrm{~cm}$ soft tissue mass lying anteriorly within the pericardium just posterior to the sternum. This was surrounded by dense calcification and compressed the right ventricle. The contents of the mass can be seen to have a similar attenuation to blood in the ventricles and are denser than the pleural effusion.

The patient was referred for surgery where extensive plate calcification external to the right ventricular outflow tract was discovered and resected. The mass was found to contain old liquefied haematoma. Postoperative recovery was uneventful with the patient reporting a gradual improvement in exertional dyspnoea.

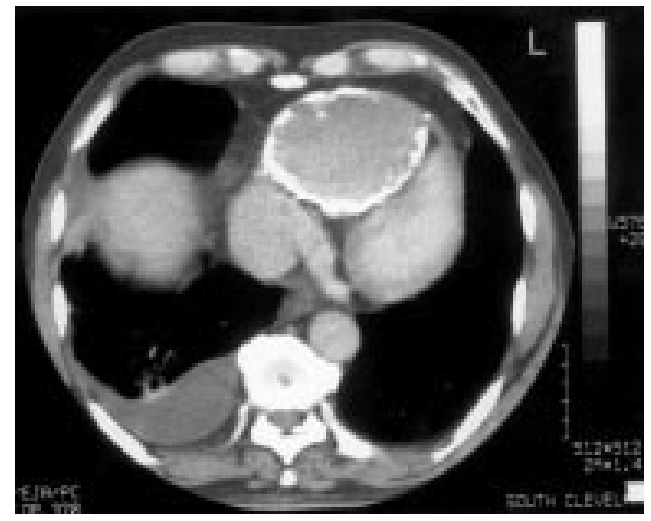

The low mortality and morbidity now associated with sternal fracture should not result in under investigation when there is clinical suspicion of associated, especially cardiac, injuries. Patients with a sternal fracture and ECG abnormalities should undergo diagnostic imaging other than just a plain chest radiograph, which is inadequate in assessing cardiac injury in this setting.

P G CAMPBELL $M$ A DE BELDER R HARTLEY mdb.sch.heart@onyxnet.co.uk 